\title{
Cross-disciplinary Concision and Clarity: Writing Social Science Abstracts in the Humanities
}

\author{
Phillip Troutman*
}

January 4, $2019^{\dagger}$

\begin{abstract}
This article details an assignment sequence asking students to apply an adaptation of Swales and Feak's (2009) model of social sciences abstract writing to articles in the humanities. This model works as an exploded diagram of the article, explicitly identifying research questions, data, methods, results, interpretations, and implications. The assignment provides students, first, with a reading tool for exposing the articulated construction of academic research articles. Second, as a writing tool, it allows students to practice comprehensive synthesis; the breakdown of multi-part claims; concision and clarity; and selective quotation. Finally, it facilitates the next step in students' research process: framing new inquiry by identifying uses and limitations in prior scholarship. This assignment sequence has been used in first-year composition and upper-division WID/WAC courses in the humanities; it can be adapted for courses in social and natural sciences and for graduate courses.
\end{abstract}

\section{Why cross-disciplinary abstracts?}

Abstracts provide a remarkably rich platform for teaching key academic writing practicesconcision, clarity, summary, paraphrase, and selective quotation-yet their variety of forms makes them difficult to model. They are not always required by humanities journals and databases (witness their absence from the MLA International Bibliography), and there remains relatively little published pedagogy or research on abstract writing in the humanities. ${ }^{1}$ By contrast, in the natural sciences and social sciences, abstracts are the norm. They are the subject of linguistic and discourse study, and abstract

\footnotetext{
*University Writing Program, the George Washington University, trout@gwu.edu. Copyright 2019 Phillip Troutman. This work is licensed under a Creative Commons Attribution-NonCommercial 4.0 International License (http://creativecommons.org/licenses/by-nc/4.0/).

${ }^{\dagger}$ Submitted, 12/20/2017; Accepted, 11/9/2018.
} 
writing is often integrated into graduate and even undergraduate pedagogy (Melander, Swales, \& Fredrickson, 1997). John Swales and Christine Feak, for example, dedicate a separate volume to it in their research writing series (Swales \& Feak, 2000, 2009). In this assignment arc, I ask students to apply an adapted version of Swales and Feak's social sciences abstract model - one which deliberately identifies research questions, methods, sources, discoveries, and multi-component claims - to articles published in the humanities.

This assignment sequence arose out of a reading comprehension problem I identified in my first-year academic writing and research course. I teach small seminars at the George Washington University in Washington, D.C.; undergraduates tend to be traditionallyaged four-year college students, with a growing number of international students, L2 learners, and first-generation college students. Reading academic journal articles across several disciplines, students could easily recognize themes or subject matter, but were less successful at identifying specific components of argument, such as relevance of prior scholarship, articulation of the research question, explanation of methods or frameworks, specific interpretation of evidence, and articulation of multi-part claims. This difficulty seemed to arise far more often in response to articles in the humanities - history, art history, cultural studies, literary studies, visual studies, philosophy - than to social science articles - sociology, psychology, anthropology, communications studies, and media studies. This may be in part because social science research articles tend to expose their structure and purpose rather explicitly, often through the IMRD format-Introduction, Methods, Results, and Discussion. Likewise, social science articles tend to deploy the three introductory moves identified by Swales and others: naming the scholarly environment/field, identifying a gap or niche in that field, and signaling how the writer/researcher will occupy that niche (Lewin, Fine, \& Young, 2001, pp. 23-61; Swales, 1990, pp. 137-166, 2004, pp. 226-234). This explicit structure, catering to disciplinary insiders, may have the added benefit of making an article's purpose and importance - its exigency - more obvious to outsiders as well, even novice readers.

By contrast, while I had chosen humanities articles with accessible arguments and approaches, the articles followed a wide variety of structures and often did not articulate the exigency of the work explicitly in the introduction. Research on academic discourse tends to confirm this impression. Fahnestock and Secor (1991), for example, find that literary scholars often establish the purpose and importance of their work only implicitly, through special topoi (e.g., by using a surface/depth binary in analyzing the meanings of a target text) that may only be recognizable to experienced readers in that field - and may not appear in the introduction. Silver and Bondi (2004) find that historians frequently submerge their own interpretative voice within the narrative language and action of their subjects, thus leaving their research questions implicit or, possibly, locating them later in the article. My own study of the interdisciplinary field of comics studies reveals that their article introductions are quite varied: only about one third use the "Swales moves"; some open with anecdotes while others simply begin narrating or analyzing, only later (or sometimes never) identifying key questions, theories, and claims (Troutman, 2010). All these practices, which may serve viable purposes for experienced disciplinary 
or interdisciplinary readers, can understandably create reading problems for readers new to those fields, especially novice academic readers.

Given this structural variety across disciplines - and my desire to have students read productively in disciplines of interest to them-I began to look for a reading tool to help students find the elements of argument wherever those elements might appear in the article. Swales and Feak's (2009) social science abstract model, adapted for the assignment sequence described below, works to accomplish this article-parsing task by forcing a full reading - and multiple readings - of the article. It can therefore address the problem identified recently by the Citation Project: students' tendency to skim articles looking only for pull quotes that seem to confirm their first impression of the article (Howard, Serviss, \& Rodrigue, 2010). This abstract model works like an exploded diagram, exposing all the article's moving parts and their relationships to one another. At the same time, it encourages students to look for what Gerald Graff (2003) has identified as productively reductive moments - places in a text where the writer synthesizes, summarizes, or translates key insights or claims into more accessible, even pithy, language. The difference here is that students are looking for those moments throughout the article, section by section, to help them break down and build back together the entire argument, not an arbitrary slice of it.

As importantly, as a writing tool, this abstract model allows students to practice a number of writing principles. Concision is the most obvious, but it often goes hand in hand with a certain brand of clarity; actor-action constructions save words and also identify the scholar's intellectual work explicitly. To effect this, I require students write in third person with what I call "intellectual action verbs" (see supplementary files). The mix of summary, paraphrase, and highly selective quotation is another skill to practice here. I use an oral exercise (described below) to help students find not only their own syntax and vocabulary whenever possible, but also those "sticky" quotations worth preserving. Successful abstracting leads to synthesis: the student's capacity to fully comprehend and recapitulate the article's argument largely in the student's own language. The abstract's brevity allows instructors to respond to larger numbers of students at once; yet its substance and specificity allow it to surface important writing issues, including mechanics and correctness, which the instructor can address on an individual basis.

I use this sequence in first-year composition and upper-division writing in the disciplines (WID) courses based largely in the humanities (with topics in visual culture, visual history, and archival research). But it could be adapted for students in the social sciences and natural sciences and for graduate courses.

\section{Assignment sequence}

My course arc generally follows a writing and research process common to the humanities: engaging in scholarly literature of topical interest to the student, identifying and framing new inquiry, conducting research to address the question, interpreting evidence to develop 
a claim, and articulating an argument in the form of an essay. This abstract assignment sequence takes place across eight class periods very early in the term, helping students learn how to engage prior scholarship and, by the end of the sequence, see how they might make smart use of it in their own research projects.

\section{Day 1: Article selection}

Students choose an article of interest from a pre-determined list. ${ }^{2}$ For each new course, I initially select ten to fifteen articles for topical and methodological variety and for the visibility of research questions, methods, discoveries, and interpretative claims. Over time, I build up more extensive lists, drawing from articles discovered by students in their subsequent research projects. I make sure all articles are full-text accessible online since students need immediate access. I keep a marked copy for myself to skim again when conferencing with students and evaluating final abstracts.

Students browse articles on the list, then discuss options in class before choosing one article to abstract. I tend to restrict them to one student per article per section, highlighting their peers as audience: Since your peers might find this article useful in their own research, what do they need to know about it? I publish excellent student examples as models on my course website, with student permission (links in supplementary files), and delete those from the choice list going forward. We carry each abstract through several stages of drafting, each framed by a new prompt discussed below.

\section{Day 2: Reading session}

First, we hold a classroom reading session on three model abstracts - one positive, two more problematic - applying my assignment criteria. Students' final abstracts must:

- restate the article's central question or topical problem.

- summarize the article's central claims, conclusions, discoveries, or results, including all components of multi-part claims.

- identify the article's primary source base in detail- e.g., genres, dates, authors, titles, of the sources analyzed.

- describe the research and/or analytical methods - e.g., theoretical approaches, key concepts, quantitative techniques, etc.

- indicate the article's larger aims, if any are stated.

In class, we read three different abstracts of the same article. One I wrote as a positive model. The other two are authentic database abstracts that prove more problematic, in that they are not comprehensive and they tend to couch claims as facts rather than interpretations or discoveries (all three models are available in supplementary files.). I read aloud my positive model while students follow along. This is their only knowledge 
of the article, so students have to trust my accuracy and comprehensiveness as we focus on the abstract's formal characteristics. Then, I ask students to work in groups to read, mark, and discuss the two database models according to my criteria.

I highlight one key caveat: the two database abstracts were not written to my assignment criteria, and they are much shorter than those I am allowing my students (94 words and 153 words, respectively, vs. 250 to 350 words in my assignment). I grant a greater word limit in part because students are novice abstract writers and need more flexibility. But I also want students to produce somewhat detailed prose they might actually use in their research essays when "coming to terms" with that prior scholarship (Harris, 2017). So, we must forgive the brevity of the database models, but they still give us a good exercise in spotting (or missing) details to include when given the space.

More importantly, these three models help reveal how prose choices can render claims more and less clear to the reader. Students routinely report that they do not see the central claims in the two database abstracts. The central claims are (mostly) there, but to reveal why students miss them, I have students underline the grammatical subject and circle the predicate of each sentence. In the database abstracts, the grammatical subjects are often long constructions of past events coupled with verbs that imply states of being and therefore factuality rather than the scholar's interpretation or discovery. In my positive model, the subject is almost always the author, Soper, and my predicates are usually Soper's actions (using my "intellectual action verbs"). I do include a passage where my grammatical subjects are the events and people from the past-Soper's subject matterbut I precede this by establishing that those past actions are all Soper's discoveries and explanations, not mere facts. My emphasis here is on making explicit the scholar's claims and contributions to the field, and Williams and Bizup's (2014) principle of actor-action sentence structure is useful in this context, both for concision and for clarity of the scholar's contribution.

\section{Day 3: Plagiarism fun! (article breakdown)}

The next task is for students to break down their chosen article in all its component parts. For this, I assign them to write a "totally plagiarized" version of an abstract, mining the article for key terms, phrases, and whole sentences, and cobbling them together with as little "glue" (and, then, etc.) as possible. My initial goal was simply to highlight and address the action of plagiarism. But more interestingly, students find forced plagiarism difficult because they are not allowed to use their own words; binding them to the author's words disallows more fluid phrasing. Moreover, students have to represent the entire article, so they must search each section for phrases and sentences that encapsulate what is going on there, and not rely solely on their first impressions.

In class, we work to de-plagiarize these abstracts in three stages. Students pair up and, without looking at any of their materials, talk to each other about their chosen articles, asking each other questions about the articles' agenda, sources, methods, concepts, discoveries, and claims for about 10 to 15 minutes. Then they spend 10 to 15 minutes 
writing, taking down as much of what they talked about from memory only, in their own language - again, without referring to any of their materials. Finally, they compare their new writing with their plagiarized abstract and look for any "sticky" terms or phrases, ones they preserved, whether inadvertently or not, from the original. We discuss those together as a group and decide which could be paraphrased and which are worth preserving as quotable nuggets. Students here use orality as a tool of writing, helping them find not only their own vocabulary and phrasing, but their own syntax as well, avoiding the "patchwriting" problem identified by the Citation Project, where students simply copy the syntax of the original, replacing individual words with rough synonyms (Howard et al., 2010).

\section{Day 4: Concision and intellectual action verbs}

Before the next class meeting, students fully draft their actual abstract, discarding the plagiarized one and starting from the one they began sketching out in class. With this first full draft, we work on concision, clarity, and what I call "intellectual action verbs." I hand out a version of Richard Lanham's "paramedic method" adapted from the Purdue OWL (see supplementary files). It focuses on prepositions and being verbs as indices of wordiness; eliminating those usually creates greater concision. Together we work through one long sample sentence, paring it down by 50 percent or more. Then they apply the paramedic method to a peer's draft abstract, first marking all prepositions and being verbs, then picking one sentence they think particularly ripe for concision. After about 15 to 20 minutes, we check in with word counts (instructors could offer a prize for largest percentage cut). There is one caveat: the cuts must preserve meaning, so there may be moments when the peer editor cannot predict the solution because the abstract writer must consult the original article for accuracy; this practice of re-reading is a valuable discovery for many students.

Next, we work on intellectual action verbs. I ask them to circle the verbs in their own abstract. States and says are relatively weak verbs here, since an abstract must load as much specific meaning as possible into each word it deploys. I hand out my "Intellectual Action Verbs" tipsheet (see supplementary files), and they work to replace non-descriptive verbs with ones more accurately capturing the action of the scholar: investigating, discovering, categorizing, etc. We talk briefly about how these verbs can shade meaning. To claim that "the article asserts . .." (vs. argues) might be read as implicit criticism. But as long as the verb usage remains "objectively" true - that we would all agree that this article asserted a claim rather than argued it - then this is fair game for this assignment. Aside from these possible shadings, the students' tone must remain neutral. The point is to accurately represent the intellectual work the author is performing in the article. 


\section{Day 5-7: Good ignorant questions (peer response)}

The final step is a series of small-group meetings ("tutorials") that replace class time, in which students and I respond to drafts. Students post drafts 24 hours before their tutorial. Peer reviewers are tasked to skim each peer's article - literally just skim it for 5 minutes to get a very rough idea what it is about - then type up a list of what I call "good ignorant questions." For example: I saw that the article analyzes some news photographs as well as propaganda posters, but you don't mention the photographs. Are they important? How are they related to the posters? Students bring all the printed material to a meeting at my office, and they lead discussion with their peers.

To minimize my preparation time, I save marked copies of all articles and skim the relevant ones before each tutorial; students list their articles when they sign up for time slots. Since students are often novice readers of this field, my feedback here is often fairly didactic, indicating aspects of the article that remain missing or mischaracterized. But I also highlight areas that are summarized well, amplify peers' questions and comments, and address choices in tone, level of quotation, and organization (following vs. rearranging the article's own structure).

\section{Day 8: But what about . . . ? (framing new inquiry)}

The abstract assignment resonates strongly with most elements of what Joseph Harris (2017) calls "coming to terms" with another text: to "define the project of the writer in your own terms" by describing its "aims," "methods," and "materials," and by identifying "keywords or passages in the text." For the purposes of the abstract, we forestall Harris's practice of "assess[ing] the uses and limits" of the text (p. 16, 20), reserving that practice as a transitional step after abstracting to help students identify new questions and realms of research they could follow in their own projects.

On the day students turn in their final abstracts, therefore, we talk explicitly about the possible uses and limitations of that prior scholarship, building a platform from which to ask new research questions. In class, students highlight one specific limitation in the article and exploit it to frame new inquiry the student could undertake. This takes the form of what I call the But what about . . .? question. Limitations may be analytical problems, but they can simply represent the justifiable parameters of the prior study, such as limitations in chronology, media sources, and subject matter focus. For example: Soper found comics artists making six distinctive uses of Irish caricatures. But what about how readers responded? Did readers see these distinctions, or see things Soper did not notice?

Students also identify potential uses of the article for their own work. These usually represent concepts, frameworks, or approaches to the research. For example: Soper creates a taxonomy of six Irish "types." But what about Chinese characters in those same newspapers? Does their different social position change their representation? Or But 
what about live action caricatures in early Hollywood films? Can Soper's comic strip taxonomy apply to moving pictures, including "talkies"?

This activity plays the abstract exercise forward to the students' own developing research interests, shows them how to make the "niche" or "gap" move Swales (2004) identifies as key to framing inquiry (pp. 227-232), and starts them in the process of applying and adapting scholarly concepts to their own analysis.

\section{Adaptations}

I have used some form of this assignment for several years, usually as the first major writing project, but sometimes as part of the research proposal. In one variation, students co-wrote in pairs to abstract three chosen articles in one single essay. By naming limitations and potential uses, they played the articles off each other in a way resembling humanities lit reviews or the book review articles published in Reviews in American History. One particularly productive adaptation would be to assign a second round of concision, cutting the final abstract to the 150 words more typical of social science journals and databases. This would raise the stakes of discernment and selection, force tough decisions about detail, and perhaps generate some creative writing solutions.

\section{Transfer and student response}

A key moment of transfer comes in students' own research proposals and research essays as they summarize one or more pieces of prior scholarship to frame their own questions. The abstract gives us a good reference point for how to bring in other work more selectively, focusing only on its relevant claims, for example, or borrowing its research methods. Students' practice in actor-action syntax can help them find ways to clarify their own project goals, research questions, methods, source uses, discoveries, and claims. Specifically, social scientists' frequent use of first person (or stand-ins like "this project") models language students can adopt or adapt, assuming the genre and discipline encourage or allow that usage.

While I have not gathered response to this assignment sequence in any systematic way, students frequently mention it positively in course evaluation surveys, even though those surveys do not ask about it directly. While students initially scoff at the notion that this is only 250-350 words, by the end they are fretting that they have only(!) 350 words to convey a complex argument. A few students have told me that this assignment helped them apply clarity and concision in other writing contexts, such as job letters, scholarship applications, and the like, where the stakes are high and you have one page to make your pitch - what a colleague of mine once called "the million-dollar letter." 


\section{Student-generated model abstracts are available on my course websites:}

- Visual Past: Images in History, https://visualpast.wordpress.com/tag/modelabstract/

- Comics Studies, http://cxstudies.blogspot.com/search/label/abstracts

\section{Assignment}

See the Supplementary Files for this article at thepromptjournal.com for a PDF facsimile of the original formatting of this assignment.

\section{Abstracts assignment sheet}

\section{the genre}

Academic researchers rely on article abstracts - summaries published in academic databases - to help them keep up with scholarship in their own field and to survey scholarship in unfamiliar fields. In the natural sciences and the social sciences, abstracts consistently follow a fairly well defined model. By contrast, in the humanities, abstracts vary widely in their format and quality. Some are written by the article's author; others by librarians or by bibliographers at the database companies who publish them. Some very clearly detail the argument, while some only hint at it or merely describe the topic. Moreover, humanities articles themselves sometimes have aims other than to argue for a single claim; they may be more exploratory or essayistic, in which case it may be difficult to abstract a discernable claim. Still, I want you to be looking for claims, as they are usually there, even if masked as fact-statements.

The language of abstracts remains neutral; abstracts do not evaluate the arguments or develop their own claims. However, for your abstracts, you may hint at strengths and limitations of the article through descriptive verbs (e.g., asserts, claims, demonstrates, suggests), as long as these terms describe accurately what the article is doing (and we would all agree with you). Write your abstract in the third person, referring to the author or authors by name and/or to the article as doing things (e.g., "the authors argue," "the article reveals," etc.). Quote only if absolutely necessary; paraphrase and summarize instead.

\section{components}

Your abstract must be 250 words (min.) to 350 words (max.) and:

- restate the article's central question or topical problem. 
- summarize the article's central claim(s), conclusions, discoveries, or results, including all components of multi-part claims.

- identify the article's primary source base in detail-e.g., genres, dates, authors, titles, of the historical images and documents they analyze.

- describe the research and/or analytical methods-e.g., theoretical approaches, key concepts, quantitative techniques, etc.

- indicate the article's larger aims, if any are stated.

Altogether, these tasks are closely related to what Joseph Harris calls coming to terms: understanding the aims, methods, and materials of another writer's work - except that you will not be using evaluative language.

\section{evaluation}

An excellent ( $A$ range) abstract accurately conveys all elements above in clear third-person prose with minimal quotation, logical organization, and close attention to the format. A strong ( $B$ range) abstract accurately conveys almost all of the elements above, with some key component of the claim or question missing or unclear, or some general lack of clarity or organization, or some serious moment of inattention to the format. A basic ( $C$ range) abstract accurately conveys the majority of the above, but with one or more major elements missing or inaccurate, or a general lack of clarity or organization, or a general inattention to the format. A poor ( $D-F$ range) abstract has major inaccuracies, profound lack of clarity or organization, or serious disregard for the format. In the $A$ to $C$ ranges, I will assign $+/$ - to reflect particular strengths or weaknesses.

\section{format \& citation}

Write your abstract in the third person, referring to the author(s) by surname(s), as in my model. The citation style here is a mixed one, modeled on those of academic book reviews in history:

- Single space, printing to one sheet.

- Title of your abstract. Use keywords from the article. Make it witty if you like.

- Give the full citation in Chicago footnote style (first name first), just below your title. Note formatting: "article title," journal title.

- Byline just below the citation: "Abstracted by Your Name Here."

- If you quote or paraphrase closely, cite page numbers in MLA style, e.g.: (171).

- Word count at bottom in square brackets, e.g.: [297 words].

\section{models}

- My model abstract: https://visualpast.wordpress.com/2014/01/14/the-irish-davechapelle-of-the-1910s-model-abstract/ 
- Student models (with permission) from past semesters of this course: https:// visualpast.wordpress.com/tag/model-abstract/

- Student models (with permission) from my course on comics and graphic novels, CXStudies: http://cxstudies.blogspot.com/?view=mosaic

\section{audience}

Your peers in this class comprise your audience. All abstracts will be available to your peers on Blackboard. Peers might peruse them for further research ideas. You are also thinking about how you would need to come to terms with this article-potentially, at least-in your research paper; so in that sense, you are an audience for the abstract.

\section{learning objectives}

You will gain practice in the principles of

- Comprehension: reading for complex, multi-part claims as well as methods, source materials, and parameters of an academic research article

- Concision: conveying meaning in a tightly constrained format

- Precision: demonstrating comprehension of an articulated academic argument

- Synthesis: using summary, paraphrase, and highly selective quotation

- Clarity: using action-oriented subject-verb structure.

* For more detail on writing social science abstracts, see John M. Swales, and Christine B. Feak, Abstracts and the Writing of Abstracts (Ann Arbor: University of Michigan Press, 2009).

Supplementary material:

- positive model abstract

- imperfect model abstracts

- plagiarism fun

- concision exercise

- intellectual action verbs 


\section{positive model abstract}

"The Irish Dave Chappelle of the 1910s?"

Kerry Soper, "From Swarthy Ape to Sympathetic Everyman and Subversive Trickster: The Development of Irish Caricature in American Comic Strips between 1890 and 1920," Journal of American Studies 39.2 (August 2005): 257-296.

Abstracted by Phillip Troutman

Soper challenges the popular and scholarly conventional wisdom that early twentiethcentury ethnic caricatures were nothing more than blatantly and demeaningly racist in intent and effect. Instead, drawing on recent studies in caricature, minstrelsy, and African-American popular culture, Soper argues that stereotypes carried meanings that were multilayered, multivalent, and unstable. He researches Irish stereotypes in newspaper and magazine comic strips between 1890 and 1920, revealing six distinctive but often overlapping categories: the "inferior, animalistic, racial type"; the "cultural scapegoat"; "the laughable ethnic fool"; "the clever or wise fool"; the "useful other' in the romantic sense"; and "the heroic, subversive trickster" (258). He closely follows three case studies - Frederick Opper's Happy Hooligan, Richard Outcault's Yellow Kid, and George McManus's Jiggs - to chronicle a trend towards greater complexity and subversion. He explains this dramatic change by way of three historical developments. First, the format evolved from single-panel gag comics to long-running multi-panel series, where character development - and therefore reader identification - were increasingly important; to sustain interest over time, readers needed to sympathize with the characters, not just laugh at them. Second, the shift from local papers to national syndication created an increasingly diverse readership, including more Irish-Americas, and comics now had to play to a broader middle-ground audience. Third, Irish-American cartoonists themselves eventually found commercial success, sometimes using Irish stereotypes to critique the dominant white culture. In his conclusion, Soper briefly addresses the larger question of whether racist caricatures can ever be fully redeemed. He critiques the "cultural amnesia or naivety" characterizing the unthinking proliferation of racial stereotypes in popular culture; he calls instead for a self-conscious "revival of playfully ambivalent genres of ethnic comedy" (296). [271 words]

\section{negative model abstracts}

Below are abstracts for the same article above from two different databases. Note that these do NOT successfully address all the criteria for your assignment. (For positive models, search the blog for "model abstracts" and click that tag to show them all. The one I wrote is on Kerry Soper's article on Irish Stereotypes in Comic Strips. Students have contributed a number of excellent abstracts I have included as models for you.) How would you grade each one below, based on the criteria laid out in the assignment sheet? What is each one doing or not doing, compared with my model above (aside from word count)? Which one of these do you like better? Why?

The first abstract lifts language directly from the article itself. This often happens when 
the author writes the abstract, and it is even seen as acceptable when database companies do it. But if you were to lift language directly in this way in your project, you would be committing plagiarism. I want you to cast your abstract in the third person (e.g., "the article argues," "the author claims," etc.) and to paraphrase and summarize, quoting as little as possible.

Kerry Soper, "From Swarthy Ape to Sympathetic Everyman and Subversive Trickster: The Development of Irish Caricature in American Comic Strips between 1890 and 1920," Journal of American Studies 39.2 (August 2005): 257-296.

abstract in Academic Search Complete database (don't follow this model)

The article focuses on the development of Irish caricature in American comic strips between 1890 and 1920. Observed from a distance, the prevalence of ethnic stereotyping in late nineteenth-century and early twentieth-century cartooning in the United States is disturbing. There were some blatantly racist depictions of ethnic minorities in cartoons and comic strips during this period, but there was also a complex spectrum of ethnic characters who played out shifting comedic and social roles. This article explores the complex patterns of identification, sympathy, and denigration that can emerge in cartoon representations of ethnic identity.

abstract in America: History and Life database (don't follow this model)

The evolution of ethnic caricature of Irish Americans in humor periodicals and newspapers during 1890-1920 reflects a softening of attitudes toward Irish immigrants as they achieved a degree of assimilation and as the public's immigration concerns began to focus on Eastern Europeans. Three popular cartoon characters, Frederick Burr Opper's Happy Hooligan, Richard Outcault's the Yellow Kid, and George McManus's Jiggs, partially retained the phenotypical stereotypes that expressed a virulent racist conception of the Irish as simian degenerates in popular comic drawings of the mid- to late 19th century, but they also invested the Irish with new, more agreeable ethnic stereotypes, portraying lovable ethnic fools and tricksters who struggled with assimilation and exposed the pretensions of the dominant society. In addition to Irish assimilation, the emergence of the Irishman as a sympathetic everyman, typified by Jiggs, also reflects changing newspaper readership, as publishers aimed to capture a larger middle-class audience, providing more genteel material. Abstract by P. Durkee. 


\section{plagiarism fun*}

Re-read: Project 1 Assignment Sheet: Abstract (Wp top menu).

Read: McBride, "'Patchwriting' is More Common than Plagiarism, Just as Dishonest," Poytner.com. [Also in Bb $>$ E-Reserves.]

\section{Sketch: printed, Double Spaced. Plagiarism Fun!}

Write a completely plagiarized abstract of your chosen article (from my Article List): Lift all the best phrases, sentences, and passages you can, cutting and pasting them into a new work document. Highlight them in the article as you find them. Don't use quotation marks. Don't cite page numbers. Don't worry about smashing them together or mixing in your own words to make full sentences. Total plagiarism.

Make it about 150-200 words. Title this document "Totally plagiarized abstract." Type up the full citation (copy if from my list if you like) and put this at the top of your plagiarized abstract. You can choose whether or not to put your name on it. Put "plagiarism" in the filename, too, so you won't mistake it later for your own prose. You stole this, remember.

Bring the sketch, PRINTED.

*This exercise takes inspiration from creative plagiarism contests, e.g., the one described in Lee Benson, "Plagiarism is the goal in hot writing contest," Deseret News, 19 Dec. 2005, https://www.deseretnews.com/article/635169845/Plagiarism-is-the-goal-in-hotwriting-contest.html.

\section{Concision \& clarity}

Concision entails deleting unnecessary words and loading as much specific meaning into the words you keep. For you as the writer, it develops discernment, making your analysis more nuanced and precise. For your readers, it helps establish clarity. It can also be worth a lot of money: grant applications impose strict word limits, and your career can be jump-started or stalled by a one-page job letter. In article abstracts, concision is essential. Do this exercise to cut unnecessary words from your abstract.*

\section{First, mark up your draft:}

1. Circle all prepositions: at, about, considering, despite, except, for, from, in, like, near, of, onto, regarding, than, that, to, upon, versus, with, etc.

2. Box all being verbs: is, are, were, will be, etc.

3. Underline the actor in each sentence (the person, idea, event, etc., doing the action), whether or not it is the grammatical subject of the sentence.

Now try to eleminate those circled/boxed words:

4. Rephrase to eliminate the prepositions, if possible. 
5. Replace being verbs with action verbs.

6. Make actors the subjects of sentences; convert verbs from passive to active voice. (This will help with clarity if not concision.)

\section{Finally, troll for words with little added value:}

7. Delete imprecise terms and phrases, e.g.: differences, changes, particular, certain, complex, based off of. Rewrite those sentences to tell us which differences, which changes, what kind of complexities, the exact nature of the relationship (causal?), etc.

8. Delete words that add little meaning: truly, actually, effectively, basically, really, etc.

9. Delete reduancies or implicit terms, e.g.: the fact that; terrible tragedy; hopes and dreams; discipline of history; future speculations; true facts; free gift.

10. Convert long phrases into shorter ones, e.g.: despite the fact that although.

*This exercise is based on Richard Lanham's "paramedic method" (summarized by the Purdue OWL, http://owl.english.purdue.edu/owl/resource/635/01/) and on Joseph Williams, Style: The Basics of Clarity and Grace, $2^{\text {nd }}$ ed., ch. 7.

\section{intellectual action verbs}

Using specific verbs to describe the intellectual work of scholars will help you identify exactly what they are doing (e.g., mentioning something is quite different from focusing on it). It can also imply your stance towards that writer's actions. E.g., if you say a scholar demonstrates something, you imply that you're convinced. If you say a scholar asserts something, however, it probably means they have not given you enough evidence or reason to be convinced. Argue and claim are usually neutral in academic contexts. The verbs below are grouped roughly by connotation - they are not synonyms. You must also use them correctly: one does not explore into a topic; one explores it. One does not ask if something is true; one asks whether it is. 


\begin{tabular}{|c|c|c|c|}
\hline exhibits & argues & questions & assumes \\
\hline reveals & claims & inquires & asserts \\
\hline conceals & reasons & explores & attests \\
\hline obscures & & investigates & proclaims \\
\hline shows & articulates & tests & expresses \\
\hline demonstrates & categorizes & & denounces \\
\hline $\begin{array}{l}\text { illustrates } \\
\text { illuminates }\end{array}$ & $\begin{array}{l}\text { differentiates } \\
\text { analyzes }\end{array}$ & $\begin{array}{l}\text { focuses on } \\
\text { delves into }\end{array}$ & instructs \\
\hline elucidates & distinguishes & spotlights & believes \\
\hline exposes & discriminates & highlights & discloses \\
\hline clarifies & chronicles & features & is certain \\
\hline explains & details & & has faith \\
\hline describes & maps & deliberates & thinks \\
\hline & graphs & ponders & feels \\
\hline discerns & composes & contemplates & doubts \\
\hline $\begin{array}{l}\text { ascertains } \\
\text { discovers }\end{array}$ & charts & meditates & is uneasy with \\
\hline finds out & defines & ruminates & judges \\
\hline sees & stipulates & imagines & valorizes \\
\hline understands & theorizes & reflects upon & values \\
\hline knows & hypothesizes & considers & privileges \\
\hline perceives & guesses & denies & \\
\hline comprehends & speculates & & [neutral/bland:] \\
\hline points out & evaluates & mentions & says \\
\hline confirms & assesses & notes & writes \\
\hline substantiates & & notices & states \\
\hline establishes & suggests & introduces & talks about \\
\hline concludes & implies & cites & conveys \\
\hline disproves & infers & ignores & communicates \\
\hline proves* & $\begin{array}{l}\text { hints } \\
\text { supposes }\end{array}$ & & discusses \\
\hline
\end{tabular}

*Note: Scholars rarely use the term prove in a formal sense. In the sciences, one can disprove a hypothesis, but never prove it. (Don't believe me? see http://theconversation.edu.au/forget--what--youve--read--science-cant--prove--a--thing--578). Math and philosophy involve proofs, but that's not the same as proving something. Lawyers might prove, but only beyond a reasonable doubt. Establishing or demonstrating are the closest we come to proving things, since we recognize that new evidence or new methods of analysis may always come to light. 


\title{
Notes
}

\begin{abstract}
${ }^{1}$ For example, of the 73 results for the tagged key term "abstract-writing" in CompPile (an important writing studies database), only two - Stotesbury (2003) and Stotesbury (2006) - analyze abstracts from the humanities (and only in comparison to those from the social and natural sciences). A few entries represent generalized abstract writing pedagogy — see Guinn (1979) and Cremmins (1996) — but the list is dominated by research and pedagogy on abstract writing in engineering, technical communication, natural sciences, and social sciences. CompPile database, WAC Clearinghouse, https://wac.colostate.edu/ comppile/search/index.cfm?pagenumber=1 (accessed 18 June 2018).
\end{abstract}

${ }^{2}$ Two of these lists are available online: "A Students' History of GW"

(https://studenthistorygw.wordpress.com/article-list/) and "The Visual Past:

Images in History“ (https://visualpast.wordpress.com/article-list/).

\section{References}

Cremmins, E. T. (1996). The art of abstracting (2nd ed.). Arlington, VA: Information Resources Press.

Fahnestock, J., \& Secor, M. (1991). The rhetoric of literary criticism. In C. Bazerman \& J. Paradis (Eds.), Textual dynamics of the professions: Historical and contemporary studies of writing in professional communities (pp. 76-96). Madison, WI: University of Wisconsin Press.

Graff, G. (2003). Clueless in academe: How schooling obscures the life of the mind. New Haven, CT: Yale University Press.

Guinn, D. M. (1979). Composing an abstract: A practical heuristic. College Composition and Communication, 30(4), 380-383.

Harris, J. (2017). Rewriting: How to do things with texts (2nd ed.). Boulder, CO: Utah State University Press.

Howard, R. M., Serviss, T., \& Rodrigue, T. K. (2010). Writing from sources, writing from sentences. Writing and Pedagogy, 2(2), 177-192.

Lewin, B. A., Fine, J., \& Young, L. (2001). Expository discourse: A genre-based approach to social science research texts. New York, NY: Continuum.

Melander, B., Swales, J. M., \& Fredrickson, K. M. (1997). Journal abstracts from three academic fields in the United States and Sweden: National or disciplinary proclivities? In A. Duszak (Ed.), Culture and styles of academic discourse (pp. 251-272). Berlin: Mouton de Gruyter.

Silver, M. S., \& Bondi, M. (2004). Weaving voices: A study of article openings in 
historical discourse. In G. Del Lungo Camiciotti \& E. Tognini Bonelli (Eds.), Academic discourse: New insights into evaluation (pp. 141-159). New York, NY: Peter Lang.

Stotesbury, H. (2003). Evaluation in research article abstracts in the narrative and hard sciences. Journal of English for Academic Purposes, 2(4), 327-341.

Stotesbury, H. (2006). Gaps and false conclusions: Criticism in research article abstracts across the disciplines. In K. Hyland \& M. Bondi (Eds.), Academic discourse across disciplines (pp. 123-148). Bern: Peter Lang.

Swales, J. M. (1990). Genre analysis: English in academic and research settings. New York, NY: Cambridge University Press.

Swales, J. M. (2004). Research genres: Exploration and applications. New York, NY: Cambridge University Press.

Swales, J. M., \& Feak, C. B. (2000). English in today's research world: A writing guide. Ann Arbor, MI: University of Michigan Press.

Swales, J. M., \& Feak, C. B. (2009). Abstracts and the writing of abstracts. Ann Arbor, MI: University of Michigan Press.

Troutman, P. (2010). The discourse of comics scholarship: A rhetorical analysis of research article introductions. International Journal of Comic Art, 12(2/3), 432-444.

Williams, J. M., \& Bizup, J. (2014). Style: The basics of clarity and grace (5th ed.). New York, NY: Pearson. 